\title{
EXPERIMENTAL VALIDATION OF AN HHO GAS CUTTING FLAME CFD MODEL
}

\author{
by Tudor Prisecaru*, Corneliu Dica**, Mihai Teodorescu**, Malina Prisecaru*, \\ Lucian Mihaescu*, Viorel Berbece*
}

\author{
* Politehnica University of Bucharest, Romania \\ ** Rokura Industrial Aplications SA, Bucharest, Romania
}

\section{Introduction}

A special patent [1] has been developed to produce hydrogen gas by means of water electrolysis. Due to the physical patented process, hydrogen is generated together with oxygen in a quasi - stoichiometric proportion realizing the $\mathrm{HHO}$ gas. An important particularity of this process consists of the mobile characteristic of the whole installation.

The main features of this productive installation are the following: gas flow rate $750 \mathrm{Nm}^{3} / \mathrm{h}$, gas pressure $0.2 \mathrm{MPa}$, gas temperature $20-45^{\circ} \mathrm{C}$.

One important field of using this gas phase mixture ( $\mathrm{HHO}$ ) can be found in cutting/welding metals and other hard materials (such as ceramic, stones, etc.), but an adequate tool has to be technically adopted in the first place.

This paper tries to establish a correlation between the unexpected visible aspect of a premixed hydrogen flame [2] generated by a normal gaseous cutting tool and the physical- kinetic mechanism involved in this process, with some conclusions concerning the best geometrical details of the cutter tool.

Briefly, a very short and condensed flame has been expected at the cutter tool outlet, but a very long and luminous flame has occurred instead - fig.1; very high temperature has been expected and low values have been registered.

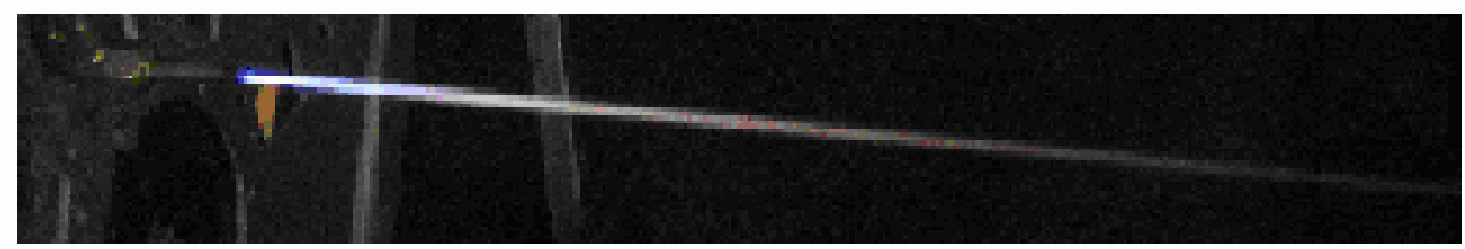

Fig.1. General aspect of the HHO flame.

Due to this fact a numerical model has been created and then an experimental session of validation has been performed in order to analyze these facts.

\section{Numerical model of the HHO flame}

To develop this numerical model of the HHO gas, Fluent code has been adopted [3]. First some important assumptions have been established:

- The HHO gas is perfectly mixed (hydrogen and oxygen) at the cutter tool outlet, due to the relative long path $(I / d)$ from the gas generator to the cutter, lap over the multitude of bents, even within the cutter;

- The HHO composition is a stoichiometric one, based upon the electrolyzing process;

- Kinetic mechanism of the HHO burning process has been adopted after Warnatz [4], as it can be analyzed in the Fig.2.

Within the Fluent case a standard $\mathrm{k}-\varepsilon$ model of turbulence has been adopted [5], considering the turbulence kinetic energy, $k$, and its rate of dissipation, $\varepsilon$, are obtained from the following transport equations:

$$
\frac{\partial}{\partial t}(\rho k)+\frac{\partial}{\partial x_{i}}\left(\rho k u_{i}\right)=\frac{\partial}{\partial x_{j}}\left[\left(\mu+\frac{\mu_{t}}{\sigma_{k}}\right) \frac{\partial k}{\partial x_{j}}\right]+G_{k}+G_{b}-\rho \epsilon-Y_{M}+S_{k}
$$

and

$$
\frac{\partial}{\partial t}(\rho \epsilon)+\frac{\partial}{\partial x_{i}}\left(\rho \epsilon u_{i}\right)=\frac{\partial}{\partial x_{j}}\left[\left(\mu+\frac{\mu_{t}}{\sigma_{\epsilon}}\right) \frac{\partial \epsilon}{\partial x_{j}}\right]+C_{1 \epsilon} \frac{\epsilon}{k}\left(G_{k}+C_{3 \epsilon} G_{b}\right)-C_{2 \epsilon} \rho \frac{\epsilon^{2}}{k}+S_{\epsilon}
$$

In these equations, $G k$ represents the generation of turbulence kinetic energy due to the mean velocity gradients, $G b$ is the generation of turbulence kinetic energy due to buoyancy, $Y M$ represents the contribution of 
the fluctuating dilatation in compressible turbulence to the overall dissipation rate and $C 1 \varepsilon, C 2 \varepsilon$, and $C 3 \varepsilon$ are constants; $\sigma_{k}$ and $\sigma_{\varepsilon}$ are the turbulent Prandtl numbers for $k$ and $\varepsilon$, respectively. Sk and $S \varepsilon$ are user-defined source terms.

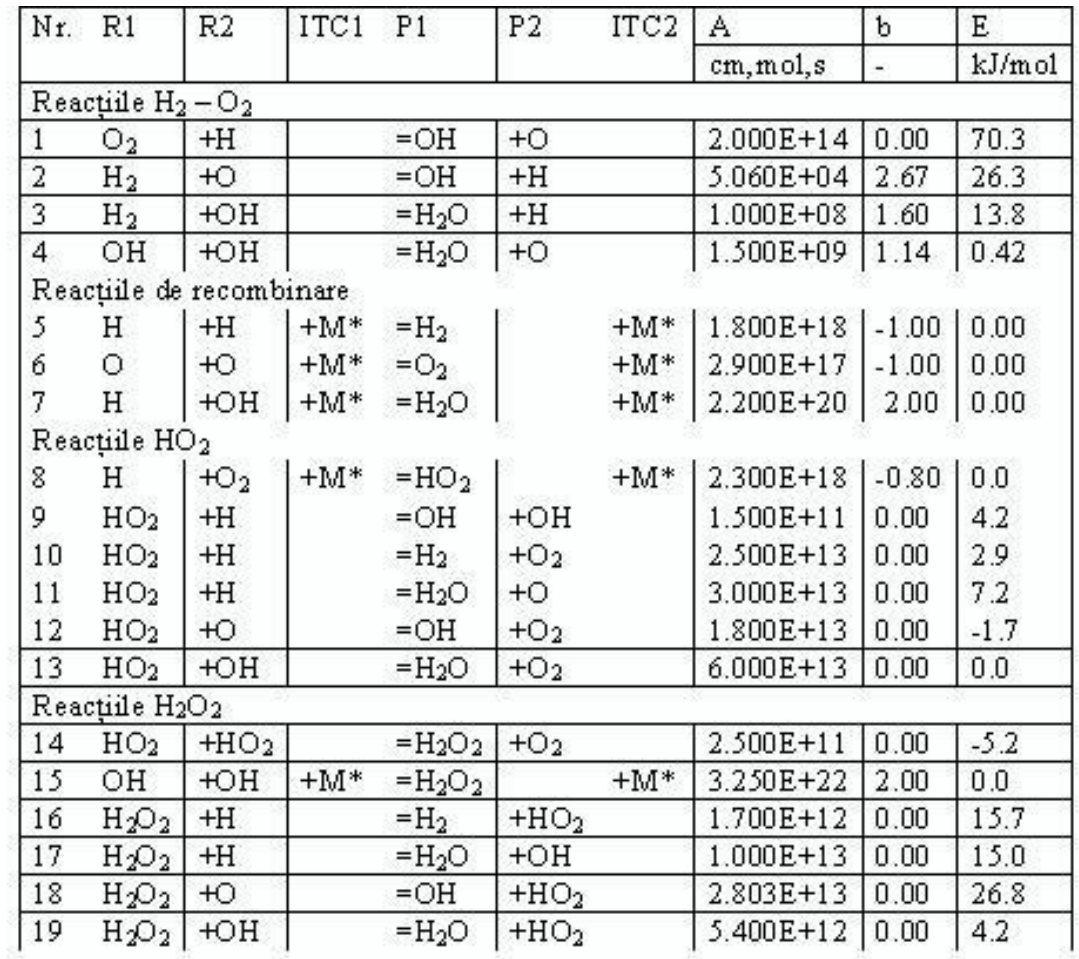

Fig.2. Synthetic presentation of the Warnatz model, for hydrogen oxidation.

The turbulent (or eddy) viscosity, $\mu t$, is computed by combining $k$ and $\varepsilon$ as follows:

$\mu_{t}=\rho C_{\mu} \frac{k^{2}}{\epsilon}$

where $C \mu$ is a constant.

The model constants $C 1 \varepsilon ; C 2 \varepsilon ; C \mu ; \sigma k$; and $\sigma_{\varepsilon}$ have the following default values [5]:

$C_{1 \epsilon}=1.44, \quad C_{2 \epsilon}=1.92, \quad C_{\mu}=0.09, \quad \sigma_{k}=1.0, \quad \sigma_{\epsilon}=1.3$

Flame post-processed aspect is presented in Fig. 3 and the temperature distribution (calculated).
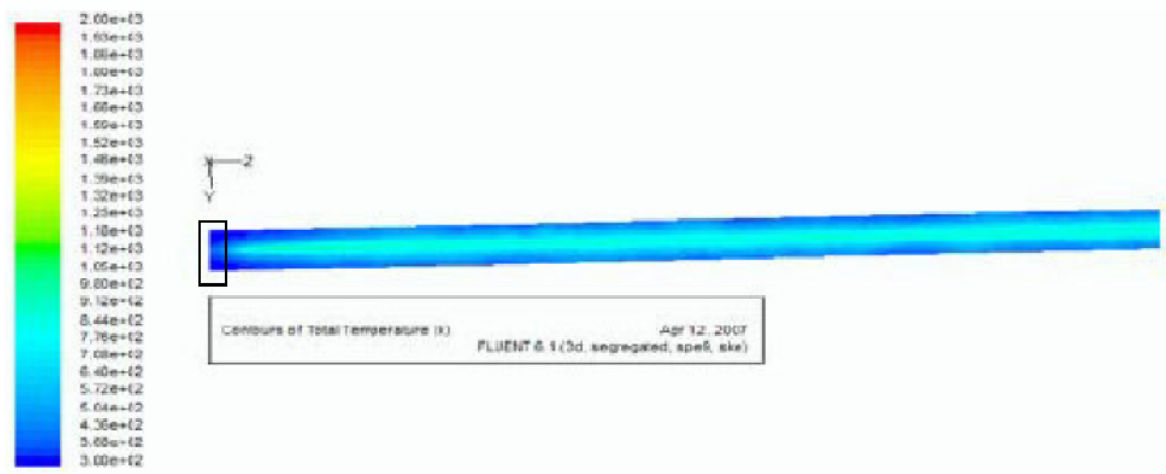

Fig. 3. Global contour of the HHO flame post-processed of a Fluent numerical simulation. 
It is to be seen that auto range temperature scale found invisible high temperatures.

Flame domain has been shorted to emphasize details as it marked.

Looking at a much smaller scale within the post-processed results we can see an interesting incipient detail, as it is shown in Fig.4.
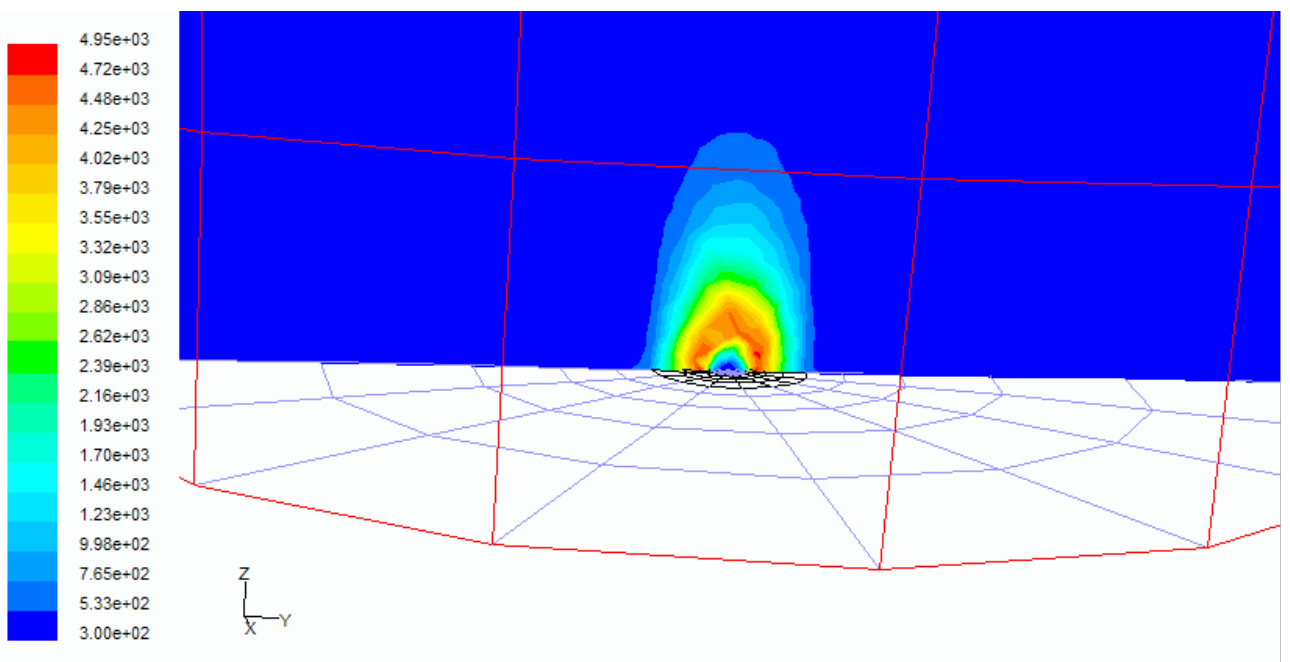

Fig.4. Detail of the incipient flame.

In this detailed figure one can see the real flame of the HHO gas, reaching the expected values of temperatures, as it can be observed on the attached Kelvin scale. This flame is a very small one, comparable with the dimensions of the nozzle. Secondary flame - observed in the fig. 3 - exists only due to the retarded recombinations mentioned by the reactions $5-19$ from the kinetic mechanism. This conclusion can be proved by the results in the fig.5, concerning the $\mathrm{HO} 2$ radicals:

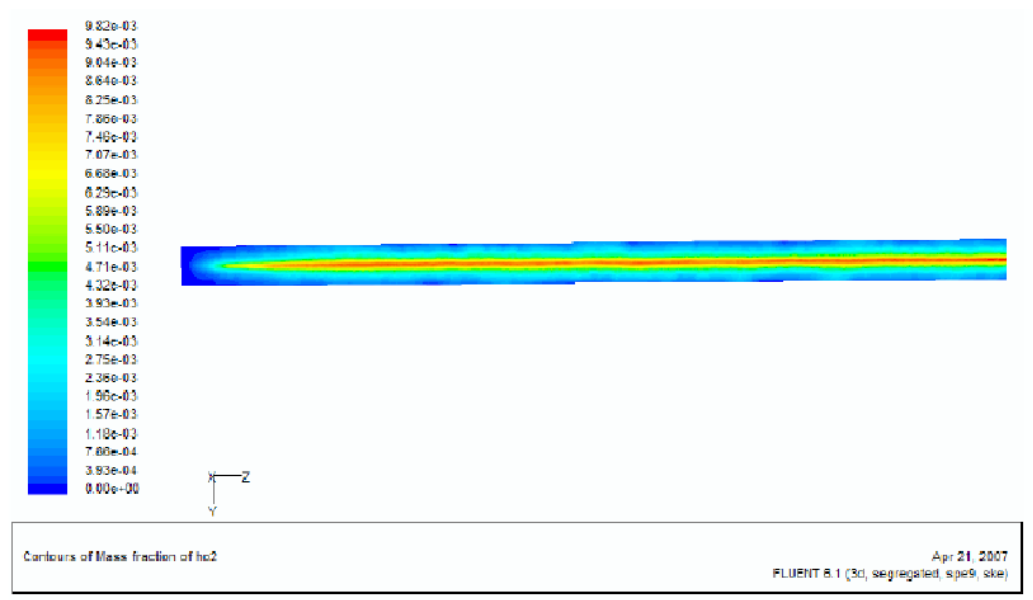

Fig.5. HO2 radical distribution inside the HHO flame.

\section{Experimental validation and conclusions}

Experimental results are presented in Fig.6. It consists of a set of temperature values recorded by a double wavelength $(0,9-1,8 \mu \mathrm{m}$ and $7-14 \mu \mathrm{m})$ infrared pyrometer OMEGA S3750 with the temperature range between $350-3000 \stackrel{\circ}{\mathrm{C}}$. 


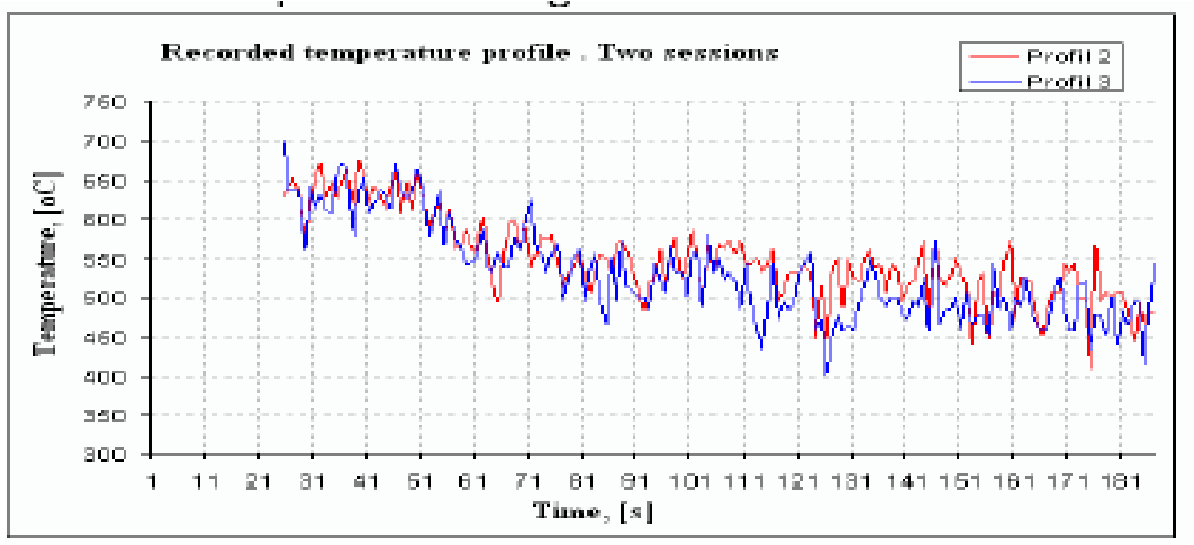

Fig.6. Recorded temperature values in two different sessions. Decreasing values due to the HHO outlet decreasing pressure.

The most important conclusion is that the $\mathrm{HHO}$ real flame is very small $(1-1.5 \mathrm{~mm})$ and the recorded and computed images are post-combustion reactions generated by the high temperatures in the main flame. Further experiments have been performed to visualize the small main flame.

All these results have been obtained by the help of the experimental installations, presented in the fig. 7 .

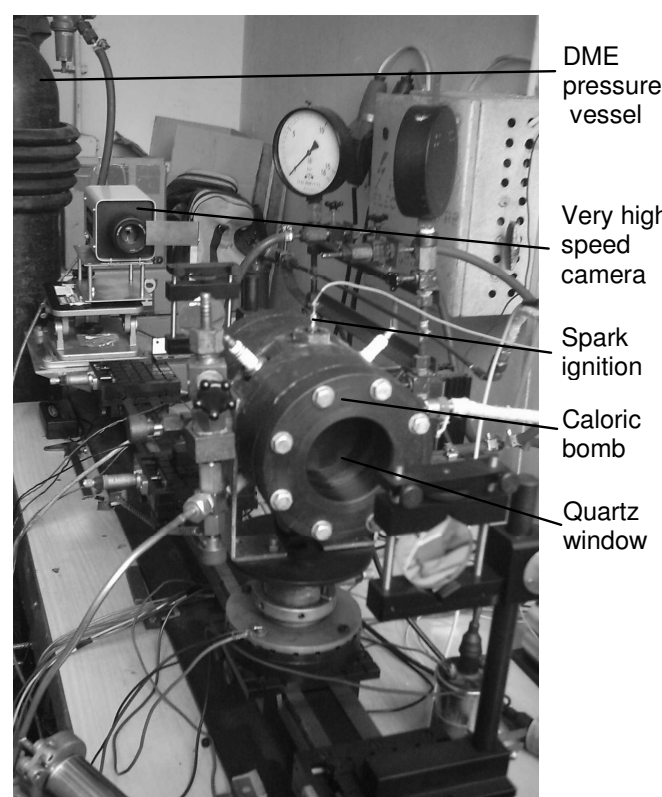

a

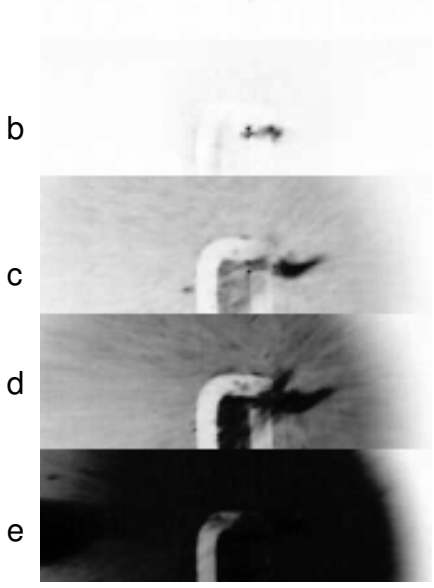

Fig.7. Experimental installation to test the ignition parameters and a series of obtained images at 16500 frames/second.

\section{Conclusions}

$>$ The flame of $\mathrm{HHO}$ gas mixture at atmospheric pressure has a special behavior due to a huge oxidation rate of hydrogen being in stoichiometric ratio and more, already premixed with oxygen. The numerical model of combustion in the primary zone of the flame point out temperature values of about $3036^{\circ} \mathrm{C}$.

$>$ Next to the primary zone, comparable as length with injection nozzle diameter, the process continue in a special space for water vapors dissociation having $10-15$ times nozzle diameter. It follows a secondary combustion zone, with bigger length, were temperature values are about $645^{\circ} \mathrm{C}$. This zone contains the gas described by Warnatz mechanism and practically constitutes the visible part of flame; the temperature of this zone is explained by its volume and the influence of oxygen that diffuses from environmental air with low caloric supply.

$>$ The research presented will help to develop some incision or adhesion advanced technologies based on $\mathrm{HHO}$ mixture ignition and combustion phenomenology. 
Acknowledgements

The authors want to express their deep gratitude to the Rokura Industrial Applications SA technical team and to the Classic and Nuclear Thermal Equipment chair technicians for their abilities during the experimental sessions.

\section{REFERENCES (selective)}

[1] D. Klein, Patent No. US 6689259 B1, Feb. 10, 2004, United States Patent.

[2] M.Chimenti, C.Di Natali, G. Mariotti, E. Paganini, G. Pieri, O. Salvetti, An IR image processing approach for characteristics combustion instability, Infrared Physics \& Technology 46 (2004) 41-47.

[3] S. Prakash, P.Y. Lee, A. Robles-Kelly Stereo techniques for 3D mapping of object surface temperature. QIRT Journal, 4 (2007), No.1, 63-85.

[4] J. Warnatz, Survey of the C/H/O reactions, Combustion Chemistry II, Springer Verlag, New York, 1991.

[5] B. E. Launder and D. B. Spalding. Lectures in Mathematical Models of Turbulence. Academic Press, London, England, 1972. 
http://dx.doi.org/10.21611/qirt.2008.06_06_08 